ERRATUM OPEN

\title{
Erratum: Ubiquitous overexpression of the DNA repair factor dPrp19 reduces DNA damage and extends Drosophila life span
}

Kathrin Garschall ${ }^{1}$, Hanna Dellago ${ }^{2}$, Martina Gáliková ${ }^{3,4}$, Markus Schosserer $\mathbb{I D}^{2}$, Thomas Flatt ${ }^{1,3}$ and Johannes Grillari ${ }^{2,5,6}$

npj Aging and Mechanisms of Disease (2017)3:10 ; doi:10.1038/s41514-017-0008-9

Erratum to: npj Aging and Mechanisms of Disease (2017); doi:10.1038/s41514-017-0005-z; Published 15 August 2017

After online publication of this article, the authors noticed an error in Fig. 2.

In panels $\mathbf{a}, \mathbf{c}$, and $\mathbf{g}$ of Fig. 2, there are the $y$-axes missing. The correct version of this figure appears below as Fig. 2.

This has now been corrected in the HTML and PDF versions of this Article.

The authors apologize for any inconvenience caused. adaptation, distribution and reproduction in any medium or format, as long as you give appropriate credit to the original author(s) and the source, provide a link to the Creative Commons license, and indicate if changes were made. The images or other third party material in this article are included in the article's Creative Commons license, unless indicated otherwise in a credit line to the material. If material is not included in the article's Creative Commons license and your intended use is not permitted by statutory regulation or exceeds the permitted use, you will need to obtain permission directly from the copyright holder. To view a copy of this license, visit http://creativecommons. org/licenses/by/4.0/.

(c) The Author(s) 2017 
a
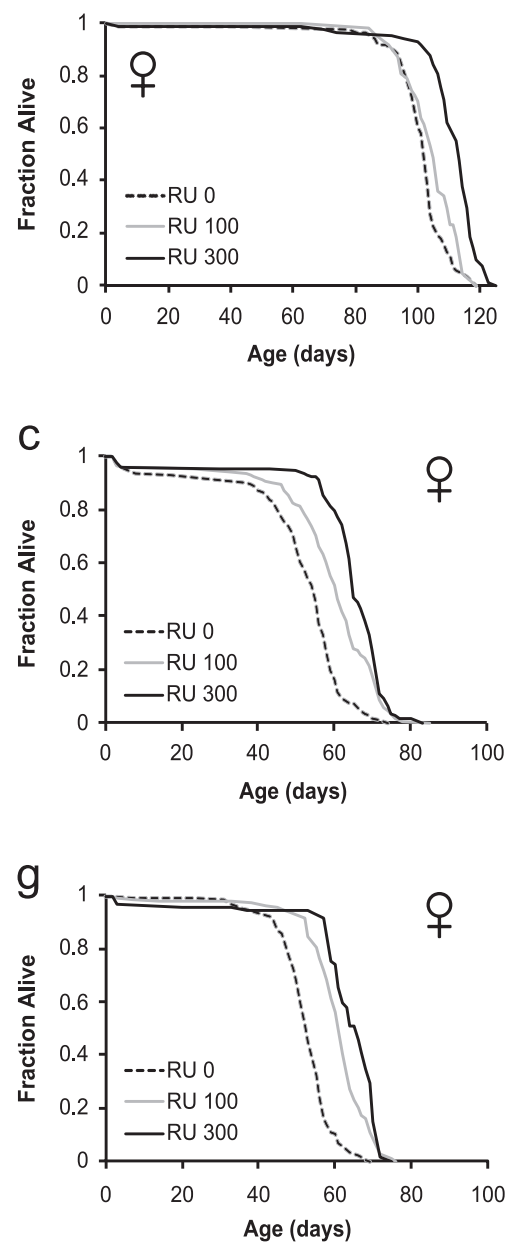

b
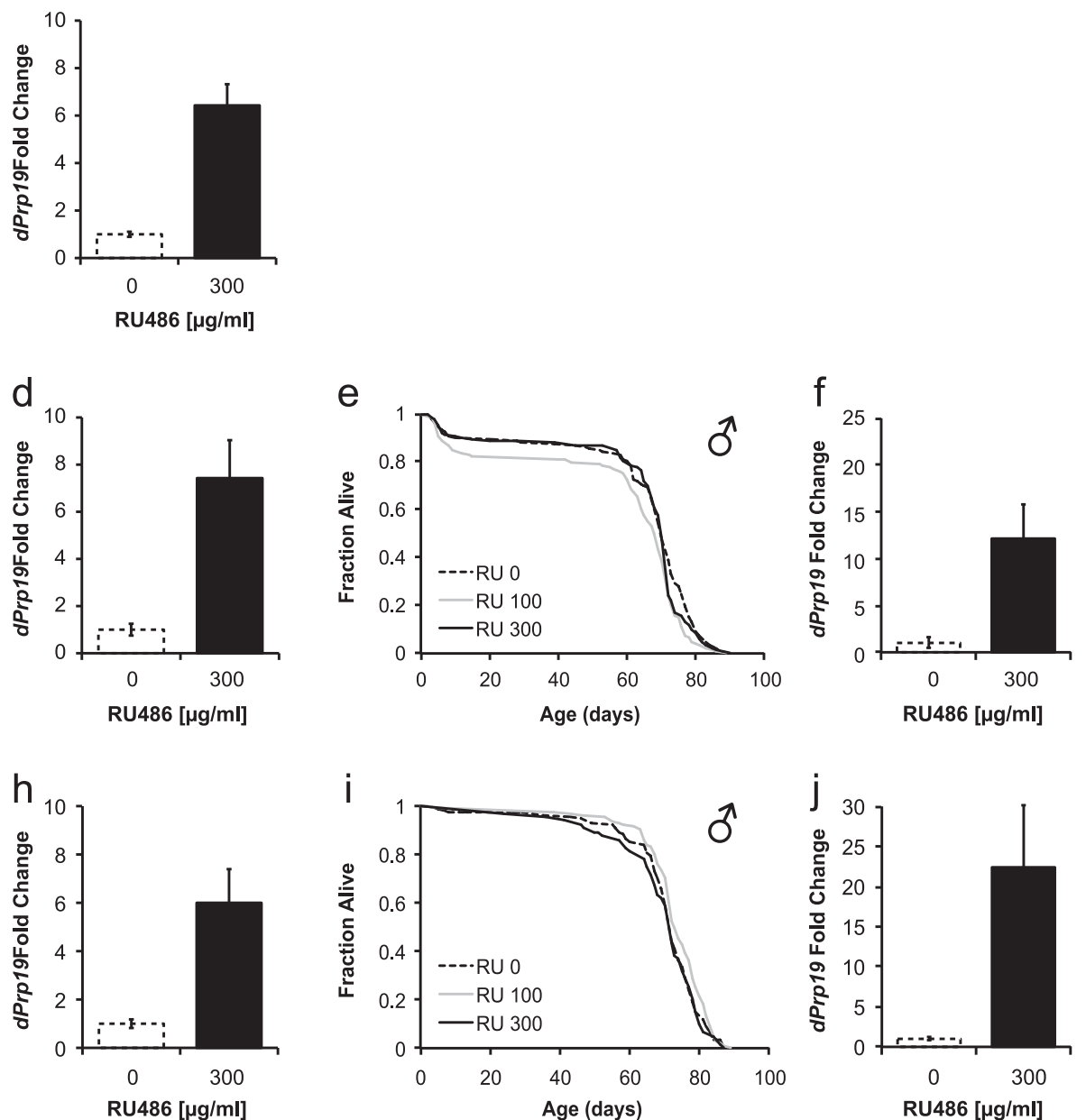

Fig. 2 Overexpression of $d \operatorname{Prp} 19$ leads to dose-dependent extension of female but not male life span. Effects of the induction of the $d P r p 19$ UAS cassette in three independent chromosomal insertions of the same transgenic construct on adult survival and $d P r p 19$ mRNA levels: a, $\mathbf{b}$ TubGS-Gal4 > UAS-dPrp19-1 (females only), c-f daGS-Gal4 > UAS-dPrp19-2(females and males), and g-j daGS-Gal4 > UAS-dPrp19-4 (females and males). a, $\mathbf{c}, \mathbf{e}, \mathbf{g}$, and $\mathbf{i}$ show survival curves of experimental flies at three concentrations of the inducer drug RU486; $\mathbf{b}$, $\mathbf{d}, \mathbf{f}, \mathbf{h}$, and $\mathbf{j}$ show quantification of $d \operatorname{Prp} 19$ expression levels relative to the $R p 49$ control after $72 \mathrm{~h}$ of exposure to $300 \mu \mathrm{g} / \mathrm{ml} \mathrm{RU} 486$. For all three overexpression constructs, we find a significant dose-dependent extension of female life span. Overall, we did not find any life span extension in males (for daGS-Gal4 > UAS-dPrp19-2 males at $100 \mu \mathrm{g} / \mathrm{ml}$ RU486 we observed a slight reduction in survival, possibly due to inadvertent "setup mortality" that might have occurred when the assay was set-up). For details of life span statistics see Supplementary Table 1 (females) and Supplementary Table 2 (males); for experimental details see Materials and Methods 